\title{
Formation and Relaxation Dynamics of Magnetic Skyrmion
}

Tae-Hoon Kim, ${ }^{1}$ Licong Peng, ${ }^{1,2}$ Ying Zhang, ${ }^{1,2}$ Liqin Ke, ${ }^{1}$ Brandt Jensen, ${ }^{1}$ X.-G. Zhang, ${ }^{3}$ Matthew J. Kramer, ${ }^{1}$ Lin Zhou $^{1 *}$

${ }^{1}$ Ames Laboratory, U.S. Department of Energy, Ames, IA, 50011, United States

${ }^{2}$ Beijing National Laboratory for Condensed Matter Physics, Institute of Physics, Chinese Academy of Sciences, Beijing 100190, China

${ }^{3}$ Department of Physics and the Quantum Theory Project, University of Florida, Gainesville, Florida 32611, United States

* Corresponding author: linzhou@ameslab.gov

Magnetic skyrmions (MS), a group of quasiparticle with topological charge, have attracted extensive research interest because they are promising candidates as information carriers in future spintronic application. MS was first detected by Mühlbauer et al.[1] with neutron scattering in bulk MnSi with a form of long-range magnetic ordered lattice-like structure. Subsequently, systematic real-space observations of skyrmion lattice (SkL) with Lorentz transmission electron microscopy (L-TEM) in B20 magnets of metallic $\mathrm{MnSi}, \mathrm{Fe}_{1-\mathrm{x}} \mathrm{Co}_{\mathrm{x}} \mathrm{Si}$, $\mathrm{FeGe}$, and insulating $\mathrm{Cu}_{2} \mathrm{OSeO}_{3}$ led to significant breakthroughs in this field.[2] Both metastable zero field and thermal dynamically equilibrium magnetic field supported skyrmions has been reported.[2] Despite extensive research in this field in the past decade, the formation and relaxation dynamics of magnetic skyrmions is still elusive. In this study, we focus on using in situ L-TEM to study dynamics of magnetic skyrmions.

Single crystals of bulk FeGe were grown by the chemical vapor transport technique. A FeGe TEM thin plate with its surface normal to the [001] crystallographic direction was prepared using a FEI Helios dual beam focused-ion-beam instrument. High resolution HAADF STEM and SAED were performed using a probe aberration-corrected FEI Titan Themis. All the defocused L-TEM images of magnetic domains were recorded using the Lorentz mode of a Tecnai F20 TEM. The quantitative in-plane magnetizations of L-TEM Fresnel images are analyzed by a phase-retrieval QPt software on the basis of the transport of intensity equation (TIE).

Figure 1a, b shows HAADF-STEM image of the high quality FeGe single crystal without discernible structural defects along [001] zone-axis. The Fe-Ge dumbbells are clearly resolved, consistent with symmetry-broken B20 structure (Fig. 1c). Transformation from spontaneous stripe domains with a modulation length of $\sim 75 \mathrm{~nm}$ (Fig. 1d) to equilibrium SkL in triangular pattern (Fig. 1e) was observed after applying a magnetic field of $87.8 \mathrm{mT}$ at $263 \mathrm{~K}$. Moreover, field cooling under the magnetic field of $87.8 \mathrm{mT}$ from $263 \mathrm{~K}$ to $96 \mathrm{~K}$ sustains the $\mathrm{SkL}$ even after the field is removed.[2]

Figure 2 summarized zero-field skyrmion evolution dynamics over temperatures, after classifying the switching process into three types according to the environment of the skyrmion, i.e. isolated skyrmions, boundary skyrmions and interior skyrmions of a SkL. For each type, the average switching time is estimated using $\tau=\mathrm{Mt} / \mathrm{N}$, where $\mathrm{N}$ is the number of switching events over the observation time interval $t$ and $M$ is the total number of skyrmion sites for the same type under observation. Other types of stripeskyrmion switching do not occur in sufficient numbers to yield reliable statistics. The relaxation time is fitted as a function of temperature using $\tau=\tau_{0} \exp \left(\frac{E_{s}}{k_{n} T}\right)$, where $\tau_{0}$ is the pre-exponential factor $\left(1 / \tau_{0}\right.$ defines the attempt frequency), $k_{R}$ is the Boltzmann constant and $E_{S}$ is the activation energy, which is 
assumed to be temperature-dependent $\left(E_{s}=C \sqrt{T_{S}-T}\right.$, where $\left.T_{S}=263 \mathrm{~K}\right)$. The summrized zero temperature activation energies $C \sqrt{T_{S}}$ is plotted in insets of Figure 2a. These results suggest nonArrhenius law behavior for zero-field skyrmion relaxation.[2] Moreover, skyrmion formation dynamics in other systems will be discussed as well [3].

References:

[1] S Mühlbauer et al. Science 323, (2009) 915.

[2] L Peng et al, Nano Letters 18, (2018) 7777.

[3] This work was supported in part by the U.S. Department of Energy, Office of Science, Basic Energy Sciences, Materials Sciences and Engineering Division. Laboratory Directed Research and Development funds through Ames Laboratory. All TEM-related work was performed using instruments in the Sensitive Instrument Facility in Ames Laboratory. Ames Laboratory is operated for the U.S. Department of Energy by Iowa State University under Contract No. DE-AC02-07CH11358.

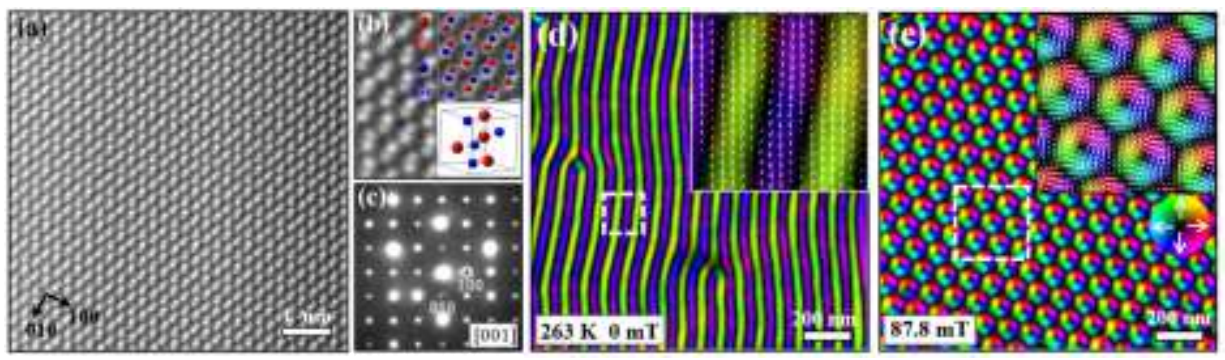

Figure 1 (a) High resolution HAADF-STEM image of FeGe taken along [001] zone axis. (b) Enlarged image from (a) revealing the Fe-Ge dumbbell in cubic B20 structure. (c) Selected area electron diffraction pattern along [001] zone axis. (d-e) Magnetic domain configuration via TIE method of (d) helical ground state and (e) skyrmion lattice at a magnetic field of $87.8 \mathrm{mT}$.[2]
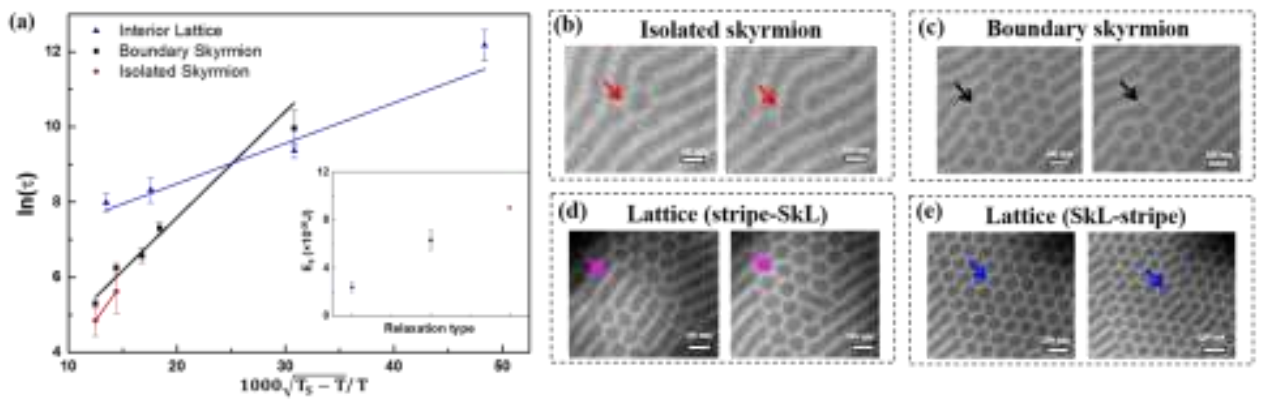

Figure 2. Relaxation dynamics of metastable zero-field skyrmions at different temperatures. (a) Linear fitting plots of $\ln (\tau)$ versus $\left(1000\left(\mathrm{~T}_{\mathrm{S}}-\mathrm{T}\right)^{1 / 2}\right) / \mathrm{T}$ for three switching processes based on direct real-space LTEM observations. The switching processes are classified into (b) Isolated skyrmion at $253 \mathrm{~K}$, (c) Boundary skyrmion at $250 \mathrm{~K}$, (d) Stripe to SkL at $243 \mathrm{~K}$, (e) skyrmion to stripe at $218 \mathrm{~K}$. The summary of corresponding energy barriers Es at $0 \mathrm{~K}$ in the insets of (a).[2] 